\title{
Exploring the Relationship Between Interpersonal Emotion Regulation and Social Anxiety Symptoms: The Mediating Role of Negative Mood Regulation Expectancies
}

\author{
Koray Akkuş ${ }^{1}$ (I) Mehmet Peker $^{1}$
}

Accepted: 11 August 2021 / Published online: 14 August 2021

(c) The Author(s), under exclusive licence to Springer Science+Business Media, LLC, part of Springer Nature 2021

\begin{abstract}
Background This study aimed to investigate the relationship between interpersonal emotion regulation (IER) and social anxiety symptoms and the mediating role of negative mood regulation expectancies (NMRE). We hypothesised that IER is positively associated with social anxiety symptoms, controlling for depression and intrapersonal emotion regulation strategies of suppression and reappraisal, and NMRE mediate this relationship.

Methods Study 1 was conducted with a student sample $(N=400)$ and Study 2 included a community sample with 271 participants.

Results Study 1 showed that, of four IER strategies, soothing and social modeling were positively, and perspective-taking was negatively related to social anxiety symptoms controlling for depression, suppression and reappraisal. Study 2 replicated these findings and extended them by showing the mediated relationship between the two IER strategies (i.e. enhancing positive affect and soothing) and social anxiety symptoms through NMRE.

Conclusions The results contribute to the limited research on IER by portraying its relationship with social anxiety symptoms and revealing the mediating role of NMRE in this relationship.
\end{abstract}

Keywords Interpersonal emotion regulation $\cdot$ Emotion regulation $\cdot$ Social anxiety $\cdot$ Negative mood regulation expectancies

\section{Introduction}

Social anxiety disorder (SAD) denotes intense and persistent fear and anxiety of being negatively evaluated in social interactions or performances. In many cases, SAD begins in early adolescence and tends to become chronic unless treated (American Psychiatric Association, 2013). Epidemiological studies show that SAD is a common disorder with a lifetime prevalence between 4 and 12\% (Crome et al., 2015; Kessler et al., 2005; Stein et al., 2017). In addition to high comorbidity rates (Koyuncu et al., 2019), SAD is associated with impairment in important areas of functioning, such as education, employment, financial status and romantic relationships (Stein \& Stein, 2008).

Koray Akkuş

koray_akkus@yahoo.com

1 Department of Psychology, Ege University, Bornova, 35030 Izmir, Turkey
While cognitive-behavioural models of SAD (e.g. Clark \& Wells, 1995; Hofmann, 2007; Rapee \& Heimberg, 1997) have predominantly emphasised cognitive (e.g. distorted beliefs, attention process) and behavioural factors (e.g. safety behaviours, avoidance), they have focused less on emotions and emotion regulation skills. However, recently, emotions and emotion regulation strategies in SAD have gained attention, and the number of studies investigating the role of emotion regulation difficulties in maintaining SAD has increased (Jazaieri et al., 2015). Several studies indicated that emotion regulation skills are indeed associated with SAD. A recent systematic review of 104 studies concluded that social anxiety is characterised by an overreliance on expressive suppression and ineffective use of cognitive reappraisal (Dryman \& Heimberg, 2018). Similarly, a meta-analysis investigating the relationship between emotion knowledge, an important component of adaptive emotion regulation, and social anxiety showed that social anxiety is negatively associated with emotion knowledge, in both its intrapersonal and interpersonal forms. Thus, individuals with social anxiety have difficulty in identifying their own and others' emotions. 
Researchers concluded that emotional knowledge may have an important role in SAD, which should be the target of treatment and interventions (O'Toole et al., 2013). Similarly, in a study evaluating new developments in cognitive-behavioural therapy of SAD, Stangier (2016) stated that emotion regulation training should be included in the treatment. In the light of the new findings, Heimberg et al. (2010, 2014) updated their original model (Rapee \& Heimberg, 1997) and included emotion dysregulation.

Although studies on emotion regulation have focused on emotion regulation as an intraindividual construct, a new conceptualisation has recently been introduced, which is called interpersonal emotion regulation (IER). Zaki and Williams (2013) suggested a comprehensive IER model, which includes both intrinsic and extrinsic and responsedependent and response-independent dimensions. In their model, while intrinsic interpersonal regulation denotes an attempt to regulate one's own emotions, efforts to regulate others' emotions constitute the process of extrinsic interpersonal regulation. Additionally, the response-dependent process requires a specific response of others. Conversely, the response-independent process occurs in social interaction contexts, regardless of the response of others. For example, expressing emotions helps regulate the negative emotions provided that others support these expressions. In contrast, labelling emotions while interacting with others may be helpful regardless of their responses. All described processes interact, and people can simultaneously use these strategies (Zaki \& Williams, 2013). Although IER is conceptualised as an adaptive process, Hofmann (2014) argued that IER strategies can be maladaptive and contribute to the maintenance of the disorder depending on the context. For anxiety disorders, IER may serve as an avoidance behaviour, which is one of the most important maintenance factors for anxiety disorders (Clark \& Beck, 2010; Hofmann, 2007). For example, Hofmann (2014) described spending too much time on social media to satisfy social needs instead of meeting people in real life or displaying anger towards others to cope with social anxiety as examples of maladaptive forms of IER. Moreover, overuse of IER can also be maladaptive, as it reduces one's sense of control on emotional experiences and makes one dependent on other people (Hofmann, 2014). Consistent with these arguments, Hofmann et al. (2016) and Koç et al. (2019) found positive correlations between social anxiety and IER strategies, such as perspective-taking, soothing and social modeling.

One of the factors that is closely related to the emotion regulation process is negative mood regulation expectancies (NMRE). Catanzaro and Mearns (1990) defined NMRE as beliefs in one's competencies in alleviating negative mood states. These beliefs have an important role in the coping process. While strong emotion regulation expectancies lead to engagement in active and adaptive responses, such as problem-solving and seeking social support, weak expectancies result in avoidance of stressors (Catanzaro \& Greenwood, 1994). Consistent with the theory, numerous studies showed that NMRE are associated with various psychological problems, such as depression (Catanzaro et al., 2014; Davis et al., 2005), anxiety (Catanzaro et al., 2000; Kassel et al., 2007) and suicide (Mrnak-Meyer et al., 2011). A recent meta-analysis also documented the relationship between NMRE and depression, anxiety, negative affect and positive affect (Mazur-Socha \& Przepiorka, 2021b).

The relationship between NMRE and emotion regulation may be bidirectional; that is, the instances of successful emotion regulation may also lead to an increase in NMRE. As individuals successfully cope with negative emotions in different situations, they may become more confident in their emotion regulation abilities. Although not as thoroughly investigated as NMRE predicting emotion regulation, several studies showed that emotion regulation skills training implemented in therapeutic interventions enhances NMRE. For example, in a sample of participants with sexual abuserelated post-traumatic stress disorder (PTSD), Cloitre et al. (2010) implemented a treatment programme that included emotion regulation training. The treatment led to improvement in the NMRE of participants, which was measured at the end of treatment and three- and six-month follow-ups. Similarly, in Cloitre et al.'s (2004) study, patients with PTSD underwent a two-phase treatment. The first phase included interpersonal and emotion regulation training. The second phase comprised actual regulation exercises through imaginal exposures. The treatment resulted in an enhancement in the NMRE ratings of patients. Contributing to the idea that coping or emotion regulation behaviours precede NMRE, Catanzaro and Greenwood (1994) reported a significant correlation between active and avoidant styles of coping and NMRE measured at a 6-week interval.

Studies investigating the relationship between NMRE and SAD are limited. An exception was the study conducted by Sung et al. (2012), which compared the relationship among NMRE, anxiety severity and quality of life between individuals with SAD and healthy controls. The study found that the clinic group had lower NMRE scores compared to the healthy control group, indicating that individuals with SAD were less likely to believe in their emotion regulation skills. Moreover, NMRE were related to the quality of life after controlling for demographic variables, comorbid diagnoses, anxiety and social anxiety symptoms in individuals with SAD (Sung et al., 2012). These results suggest that NMRE play an important role in emotion regulation processes.

Few studies (e.g. Hofmann et al., 2016; Koç et al., 2019) and discussions (Hofmann, 2014) suggest that IER is associated with SAD; however, the contribution of IER to SAD symptoms and intermediary processes between IER and SAD relationship have yet to be examined. To this aim, we 
report two studies. In the first study, we examine the relationship between IER strategies and SAD. Since SAD is associated with depression (Acarturk et al., 2008; Beesdo et al., 2007) and intrapersonal emotion regulation (Jazaieri et al., 2015; Spokas, et al., 2009), these variables are controlled to investigate the relationship between IER and SAD. Thus, the first study aims to examine IER's role on SAD accounting for depression and intrapersonal emotion regulation strategies. We hypothesise that IER strategies are positively associated with SAD, controlling for depression and two intrapersonal emotion regulation strategies: suppression and reappraisal (Gross \& John, 2003).

The second study aims to replicate the findings of Study 1 in a community sample and tests a model that examines the mediating role of NMRE on the relationship between IER and social anxiety symptoms. Hofmann (2014) argued that overreliance on IER strategies to cope with negative experiences may reduce one's confidence and control over the emotional experience. Repetitive and frequent regulation of emotions through others can bring about perceptions that one is ineffective at regulating emotions on their own, which in turn contribute to the maintenance of symptoms (Hofmann et al., 2016). Since NMRE pertain to people's beliefs about their ability to effectively cope with negative emotions, they are relevant the process depicted by Hofmann et al. Frequently resorting to IER strategies may be a maintaining factor for SAD because it may diminish one's expectancies about their own emotion regulation abilities. Thus, seeking others for emotion regulation may prevent processes that would otherwise build one's confidence in own emotion regulation skills (e.g. NMRE) and contribute to the maintenance of social anxiety symptoms. Following similar reasoning, Ray-Yol and Altan-Atalay (2020) investigated the role of NMRE in the IER-psychological distress relationship. They found that NMRE have a mediating role in the relationship between the soothing dimension of IER and psychological distress. The authors contended that individuals who seek others to cope with negative emotions may become too reliant on others and fail to build confidence in their abilities to handle unpleasant emotions. Based on these, we hypothesise that IER strategies are negatively related to NMRE and NMRE are negatively associated with SAD symptoms. Moreover, we hypothesise that IER strategies are positively associated with SAD symptoms both directly and through the mediation of NMRE.

\section{Study 1 Methods}

\section{Participants and Procedure}

Psychology and sociology undergraduates who were enrolled in psychology classes at a large public university in the western region of Turkey were included in the study. Initially, we received 422 completed questionnaires; however, after the deletion of duplicate submissions and outliers, we had a final sample of 400 participants. Of the 400 participants, $345(86.3 \%)$ were women, $53(13.3 \%)$ were men and $2(0.5 \%)$ reported non-binary gender categories. The average age was 20.28 years $(S D=1.29)$. The questionnaire was administered via an online tool, and participants were offered course credit in exchange for their participation. Before filling out the questionnaires, participants provided informed consent. The questionnaires took approximately 25 min to complete.

\section{Instruments}

\section{Social Anxiety}

We measured the social anxiety symptoms of participants using the Liebowitz Social Anxiety Scale (LSAS) developed by Liebowitz (1987) and translated into the Turkish by Soykan et al. (2003). The scale consists of 24 items, which are rated for fear and avoidance experienced in performance- and social interaction-related situations separately (e.g. 'meeting strangers'). The responses were rated on a four-point scale, ranging from $0=$ none to $3=$ severe for fear and $0=$ never to $3=$ usually for avoidance. The scale demonstrated good psychometric qualities with a total reliability score of 0.96 (Heimberg et al., 1999).

\section{Interpersonal Emotion Regulation}

Participants rated their agreement with the IER strategies using the scale developed by Hofmann et al. (2016) and translated into the Turkish by Gökdağ et al. (2019). The 20 -item scale captured four five-item subscales, namely, enhancing positive affect (e.g. 'I like being around others when I'm excited to share my joy', $\alpha=0.89$ ), perspectivetaking (e.g. 'Having people remind me that others are worse off helps me when I'm upset', $\alpha=0.91$ ), soothing (e.g. 'I look to others for comfort when I feel upset', $\alpha=0.94$ ) and social modeling (e.g. 'It makes me feel better to learn how others dealt with their emotions', $\alpha=0.93$ ). The scale is rated on a five-point scale ranging from $1=$ not true for me at all to $5=$ extremely true for me.

\section{Control Variables}

We controlled for several theoretically relevant variables to account for their effect in our analyses. These included depression and intrapersonal emotion regulation strategies, which are related to SAD (Ledley et al., 2005; Werner et al., 2011). Depression was assessed with the Hospital Anxiety and Depression scale developed by Zigmond and Snaith 
(1983) and translated into the Turkishby Aydemir et al. (1997). The depression subscale consists of seven items (e.g. 'I still enjoy the things I used to enjoy') rated on a four-point scale that has different response categories for each question (e.g. $0=$ most of the time, $3=$ not at all). The depression subscale has been shown to have good reliability with a mean score of 0.82 across studies (Bjelland et al., 2002). Intrapersonal emotion regulation was measured with a scale developed by Gross and John (2003) and translated into the Turkishby Ulaşan-Özgüle and Sümer (2017). The scale has two dimensions that capture reappraisal (e.g. 'I control my emotions by changing the way I think about the situation I'm in') and suppression (e.g. 'I control my emotions by not expressing them'), factors that were rated on a sevenpoint scale ranging from $1=$ strongly disagree to $7=$ strongly agree. The reported reliability estimates ranged from 0.75 to 0.82 for reappraisal and 0.68 to 0.76 for suppression across four studies (Gross \& John, 2003).

\section{Data Analysis}

Analyses were conducted using SPSS version 26. Before the data analysis, we checked the data for outliers by estimating Mahalanobis distances. Five multivariate outlier cases with significant distance values $(p<0.001)$ were removed from the dataset. We screened the skewness and kurtosis values of the variables for deviations from normality. The skewness values ranged from -1.09 to 0.68 , and the kurtosis values ranged from -0.97 to 1.05 , which were acceptable. We examined the variance inflation factors of the independent and control variables to screen for multicollinearity and found that they were all $<3$, with the highest value being 1.78. We conducted a hierarchical multiple regression analysis to test the hypotheses. In the first step, we regressed social anxiety on depression, reappraisal, suppression and in the second step, we added the four IER strategies.

\section{Study 1 Results}

Table 1 provides the means, standard deviations, bivariate correlations between the variables, and reliability estimates. The hierarchical regression analysis results were provided in Table 2 . In the first step, depression $(\beta=0.23$, $p<0.001)$ and suppression $(\beta=0.12, p=0.02)$ were both significantly and positively related to social anxiety symptoms, while reappraisal $(\beta=-0.02, p=0.76)$ was not. The variables explained $8 \%$ of the variance in social anxiety symptoms. In the second step, four IER strategies were included in the equation. The inclusion of IER strategies significantly improved the model $(\Delta F=11.28, p<0.001)$. Of the four IER strategies, perspective-taking $(\beta=-0.12$, $p=0.04$ ) was negatively related to social anxiety symptoms, while soothing $(\beta=0.20, p=0.001)$ and social modeling $(\beta=0.23, p<0.001)$ were positively associated with social anxiety symptoms. Enhancing positive affect was not significantly associated with social anxiety symptoms $(\beta=-0.01$, $p=0.82$ ). The second equation explained $18 \%$ variance, by incrementing an additional $10 \%$.

We tested the relationship between IER and social anxiety symptoms among participants who had an LSAS score of 50 or above to explore the relationships at clinically relevant values of LSAS. The LSAS cut-off score of 50 provided a high hit rate $(95.5 \%)$ and low false-negative rate $(4.5 \%)$ among individuals with generalised social phobia and was suggested by Soykan et al. (2003) in Turkish contexts. For individuals who had an LSAS score $\geq 50(N=212)$, the estimates were mostly similar to those obtained for the whole sample. Soothing $(\beta=0.18, p=0.03)$ and social modeling ( $\beta=0.25, p=0.004)$ were significantly related to social anxiety symptoms, while enhancing positive affect $(\beta=-0.04$, $p=0.55$ ) was not. The only difference was the perspectivetaking-social anxiety symptoms relationship $(\beta=-0.04$, $p=0.62$ ), which was not significant.
Table 1 Descriptive statistics, reliability estimates and correlations among Study 1 variables

\begin{tabular}{lcccccccc}
\hline Variable & 1 & 2 & 3 & 4 & 5 & 6 & 7 & 8 \\
\hline 1. Social anxiety & $(0.96)$ & & & & & & & \\
2. Enhancing positive affect & 0.08 & $(0.88)$ & & & & & & \\
3. Perspective-taking & 0.06 & $0.17^{* *}$ & $(0.78)$ & & & & & \\
4. Soothing & $0.24^{* *}$ & $0.37^{* *}$ & $0.45^{* *}$ & $(0.90)$ & & & & \\
5. Social modeling & $0.22^{* *}$ & $0.41^{* *}$ & $0.52^{* *}$ & $0.51^{* *}$ & $(0.88)$ & & & \\
6. Depression & $0.26^{* *}-0.12^{*}$ & $-0.11^{*}$ & 0.07 & -0.08 & $(0.76)$ & & \\
7. Reappraisal & -0.05 & $0.22^{* *}$ & $0.32^{* *}$ & 0.08 & $0.30^{* *}-0.18^{* *}$ & $(0.83)$ & \\
8. Suppression & $0.18^{* *}-0.10^{*}$ & 0.09 & $-0.12^{*}$ & -0.04 & $0.27 * *$ & 0.09 & $(0.75)$ \\
Mean & 51.84 & 21.16 & 9.13 & 14.90 & 19.81 & 8.72 & 21.90 & 13.19 \\
$S D$ & 26.14 & 3.77 & 3.75 & 5.51 & 5.43 & 3.87 & 5.90 & 4.46 \\
\hline
\end{tabular}

$N=400$. Reliability estimates were reported in parentheses on the diagonal $* p<0.05, * * p<0.01$ 
Table 2 Hierarchical multiple regression analysis results of the student sample

\begin{tabular}{|c|c|c|c|c|c|c|c|c|}
\hline \multirow[t]{2}{*}{ Variable } & \multicolumn{4}{|l|}{ Step 1} & \multicolumn{4}{|l|}{ Step 2} \\
\hline & $b$ & $S E$ & $\beta$ & $t$ & $b$ & $S E$ & $\beta$ & $t$ \\
\hline Depression & 1.54 & 0.35 & 0.23 & $4.45 * * *$ & 1.30 & 0.34 & 0.19 & $3.84 * * *$ \\
\hline Reappraisal & -0.07 & 0.22 & -0.02 & -0.31 & -0.32 & 0.23 & -0.07 & -1.43 \\
\hline Suppression & 0.71 & 0.30 & 0.12 & $2.41 *$ & 1.05 & 0.29 & 0.18 & $3.62 * * *$ \\
\hline Enhancing positive affect & & & & & -0.09 & 0.37 & -0.01 & -0.23 \\
\hline Perspective-taking & & & & & -0.85 & 0.41 & -0.12 & $-2.06^{*}$ \\
\hline Soothing & & & & & 0.93 & 0.28 & 0.20 & $3.30 * *$ \\
\hline Social modeling & & & & & 1.13 & 0.29 & 0.23 & $3.83^{* * *}$ \\
\hline$R^{2}$ & & & 0.08 & & & & 0.18 & \\
\hline$\Delta R^{2}$ & & & & & & & 0.10 & \\
\hline
\end{tabular}

\section{Study 2 Methods}

\section{Participants and Procedure}

The online survey link was distributed via announcements posted on social media (e.g. Instagram) accounts of authors and their acquaintances to recruit participants. The study was carried out in accordance with principles and standards of Helsinki Declaration. Participants provided informed consent before filling out the survey and did not receive any compensation for participation. Initially, we received 282 completed surveys. Deletion of unusable surveys due to duplicate responses and outliers resulted in 271 participants. The sample had a mean age of 35.38 years $(S D=11.84), 173$ (63.8\%) were women, and 98 (36.2\%) were men. A majority of the sample were university graduates $(N=174,64.2 \%)$, $46(17 \%)$ had graduated from high school or below, and 51 $(18.8 \%)$ had received graduate degrees. Of the participants, $164(60.5 \%)$ were either married or in a relationship, and 107 (39.5\%) were single.

\section{Instruments}

\section{Negative Mood Regulation Expectancies}

In addition to the instruments presented in Study 1, we used the NMRE Scale developed by Catanzaro and Mearns (1990) and translated into the Turkish by Kaymakçıoğlu (2001). The scale assesses people's beliefs in their behaviours and cognition that regulate negative mood states. It has 30 items (e.g. 'When I'm upset, I believe that I can do something to feel better', 'When I'm upset, I believe that I can feel better by doing something creative') that are rated on a five-point scale ranging from $1=$ strongly disagree to $5=$ strongly agree. In the study of Catanzaro and Mearns (1990), the Cronbach alpha estimates ranged from 0.87 to
0.92. The original scale has been shown to be distinct from social desirability and locus of control and a predictor of depression and dysphoria, which indicated construct validity. The Turkish translation had a Cronbach alpha value of 0.88 . It demonstrated expected relationships with depression, anxiety and coping strategies.

\section{Data Analysis}

The analyses were performed using SPSS version 26 and Mplus 8 (Muthén \& Muthén, 2017). We applied the same screening procedure as in Study 1 and removed four outlier cases based on significant Mahalanobis distance values $(p<0.001)$. The skewness values of the variables ranged from -1.04 to 0.66 , and kurtosis values ranged from -0.68 to 0.92 . We examined variance inflation factors to check for multicollinearity and found that the highest value was 1.91. To test the mediating role of NMRE on the relationship between IER strategies and SAD, we run a single path analysis that included all variables. The significance of mediated paths was tested with bootstrapping procedure by generating 5000 bootstrapped resamples to obtain $95 \%$ bias-corrected confidence intervals.

We employed direction dependence analysis (DDA) to assess the direction of the relationship between IER strategies and NMRE. DDA is a framework that is employed to confirm or disconfirm the ordering of a relationship by examining several properties of cross-sectional data. Specifically, (a) distributional characteristics (e.g. kurtosis, skewness) of variables, (b) distributional properties of residuals of competing models, and (c) independence of predictors and errors are examined. It was stated that in an accurately specified model, (a) the dependent variable will be closer to the normality, (b) the residual will be closer to normality, and (c) assumption of independence will be confirmed compared to an inaccurately specified model (Wiedermann 
\& Sebastian, 2019; Wiedermann \& von Eye, 2015; Wiedermann et al., 2019, 2020). These properties were investigated for a model where four IER strategies precede NMRE (IER $\rightarrow$ NMRE) and a reversed model where NMRE precede the IER strategies (NMRE $\rightarrow$ IER) to infer the ordering of the relationship.

\section{Study 2 Results}

Table 3 shows descriptive statistics, Cronbach alpha estimates, and correlations among Study 2 variables. As a first step, we repeated the hierarchical multiple regression analysis performed in Study 1 to investigate whether the results are comparable with a community sample. The hierarchical regression analysis results are provided in Table 4 . The results obtained in Study 1 were largely confirmed.
Perspective-taking $(\beta=-0.17, p=0.01)$, soothing $(\beta=0.23$, $p=0.003)$ and social modeling $(\beta=0.18, p=0.02)$ were significantly related to social anxiety symptoms. The model explained $17 \%$ of the variance in social anxiety symptoms, and the inclusion of IER strategies significantly improved the model $(\Delta F=5.39, p<0.001)$. Overall, these results demonstrate the robustness of findings across two samples.

In the path analysis, we simultaneously estimated all hypothesised relationships. Depression, reappraisal, and suppression were added as control variables for social anxiety symptoms and NMRE, which resulted in an estimation of a saturated model. The relationships and standardised path estimates are shown in Fig. 1. Of the control variables, depression was significantly associated with social anxiety symptoms $(\beta=0.14, p=0.03)$ and NMRE $(\beta=-0.38, p<0.001)$; reappraisal was not significantly related to social anxiety symptoms $(\beta=0.05, p=0.46)$ and

Table 3 Descriptive statistics, reliability estimates, and correlations among Study 2 variables

\begin{tabular}{|c|c|c|c|c|c|c|c|c|c|}
\hline Variable & 1 & 2 & 3 & 4 & 5 & 6 & 7 & 8 & 9 \\
\hline 1. Social anxiety & $(0.96)$ & & & & & & & & \\
\hline 2. Enhancing positive affect & -0.00 & $(0.90)$ & & & & & & & \\
\hline 3. Perspective-taking & 0.01 & $0.24 * *$ & $(0.79)$ & & & & & & \\
\hline 4. Soothing & $0.22 * *$ & $0.44 * *$ & $0.53 * *$ & $(0.86)$ & & & & & \\
\hline 5. Social modeling & $0.16^{* *}$ & $0.48 * *$ & $0.52 * *$ & $0.57 * *$ & $(0.87)$ & & & & \\
\hline 6. Depression & $0.30 * *$ & $-0.14 *$ & -0.04 & 0.11 & -0.05 & $(0.77)$ & & & \\
\hline 7. Reappraisal & -0.06 & $0.35 * *$ & $0.14 *$ & 0.11 & $0.29 * *$ & $-0.26 * *$ & $(0.91)$ & & \\
\hline 8. Suppression & $0.15^{*}$ & 0.07 & 0.11 & 0.06 & 0.09 & $0.12 *$ & $0.20 * *$ & $(0.79)$ & \\
\hline $\begin{array}{l}\text { 9. Negative mood regulation } \\
\text { expectancies }\end{array}$ & $-0.35^{* *}$ & $0.23 * *$ & 0.05 & $-0.13^{*}$ & 0.11 & $-0.54 * *$ & $0.45^{* *}$ & -0.11 & $(0.88)$ \\
\hline Mean & 40.22 & 20.18 & 10.58 & 14.49 & 19.16 & 7.24 & 22.88 & 12.65 & 105.08 \\
\hline$S D$ & 25.48 & 4.23 & 3.72 & 4.94 & 5.46 & 3.84 & 6.84 & 4.81 & 16.30 \\
\hline
\end{tabular}

$N=271$. Reliability estimates were reported in parentheses on the diagonal

${ }^{*} p<0.05, * * p<0.01$

Table 4 Hierarchical multiple regression analysis results of the community sample

\begin{tabular}{|c|c|c|c|c|c|c|c|c|}
\hline \multirow[t]{2}{*}{ Variable } & \multicolumn{4}{|l|}{ Step 1} & \multicolumn{4}{|l|}{ Step 2} \\
\hline & $b$ & $S E$ & $\beta$ & $t$ & $b$ & $S E$ & $\beta$ & $t$ \\
\hline Depression & 1.90 & 0.40 & 0.29 & $4.71 * * *$ & 1.60 & 0.40 & 0.24 & $3.98 * * *$ \\
\hline Reappraisal & -0.05 & 0.23 & -0.01 & -0.21 & -0.15 & 0.24 & -0.04 & -0.65 \\
\hline Suppression & 0.64 & 0.32 & 0.12 & $2.01 *$ & 0.68 & 0.31 & 0.13 & $2.20 *$ \\
\hline Enhancing positive affect & & & & & -0.66 & 0.42 & -0.11 & -1.58 \\
\hline Perspective-taking & & & & & -1.21 & 0.48 & -0.18 & $-2.51^{*}$ \\
\hline Soothing & & & & & 1.20 & 0.40 & 0.23 & $3.01 * *$ \\
\hline Social modeling & & & & & 0.84 & 0.35 & 0.18 & $2.36^{*}$ \\
\hline$R^{2}$ & & & 0.11 & & & & 0.17 & \\
\hline$\Delta R^{2}$ & & & & & & & 0.06 & \\
\hline
\end{tabular}

$N=271$. Unstandardised and standardised estimates were reported

$S E$ standard error

$* p<0.05, * * p<0.01, * * * p<0.001$ 
Fig. 1 Path analysis results

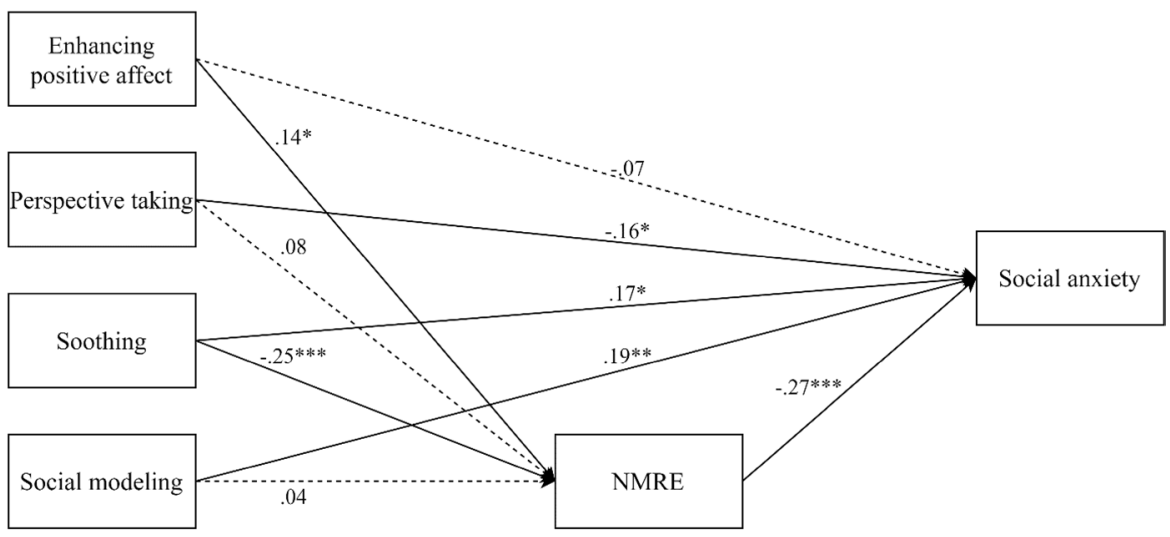

Note. $N=271$. Standardised values were reported. The dashed lines represent nonsignificant paths. The control variables are not shown in the figure for brevity. NMRE: negative mood regulation expectancies.

$* p<.05, * * p<.01, * * * p<.001$.

was significantly related to NMRE $(\beta=0.33, p<0.001)$; and suppression was not significantly related to social anxiety symptoms $(\beta=0.09, p=0.12)$ and was significantly associated with NMRE $(\beta=-0.14, p=0.01)$. NMRE were negatively and significantly related to social anxiety symptoms $(\beta=-0.27, p<0.001)$. The IER strategies of enhancing positive affect $(\beta=0.14, p=0.01)$ and soothing $(\beta=-0.25, p<0.001)$ were significantly related to NMRE. Of the IER strategies, perspective taking $(\beta=-0.16, p=0.02)$, soothing $(\beta=0.17, p=0.03)$, and modelling $(\beta=0.19, p=0.01)$ were significantly associated with social anxiety symptoms. The model explained $21 \%$ of the variance in social anxiety and $45 \%$ of the variance in NMRE. The results showed that the indirect relationship between enhancing positive affect and social anxiety symptoms through NMRE was significant, coefficient $=-0.23, S E=0.11,95 \%$ CI $[-0.50,-0.05]$. Likewise, the indirect relationship between soothing and social anxiety through NMRE was also significant, coefficient $=0.34, S E=0.15,95 \%$ CI [0.12, 0.72].

We examined whether removing the control variables changes the direction or significance of estimates by running the path analysis without them. There was no change in either direction or significance of the paths and mediation results, indicating the robustness of the estimates. Taken together, the results provide partial support for mediation hypotheses. The mediated relationships of enhancing positive affect and soothing with social anxiety symptoms through NMRE were significant, while the mediated relationships of perspective-taking and social modeling with social anxiety symptoms through NMRE were not.

\section{Alternative Model Tests}

Previous research has documented that NMRE is a predictor of coping and emotion regulation (Catanzaro \& Mearns, 1999; Mazur-Socha \& Przepiórka, 2021a). Thus, NMRE may affect the endorsement and use of different IER strategies, which in turn may alter social anxiety symptoms. To address this possibility, we compared an alternative mediation model in which four IER strategies mediate the relationship between NMRE and social anxiety symptoms with the hypothesised model. We investigated how much variance the two models without the mediators accounted for in social anxiety symptoms and then compared the contribution of mediators by examining the change in $R^{2}$ when the mediators are included. In the hypothesised model, four IER strategies and control variables explained $17 \%$ variance in social anxiety symptoms and inclusion of NMRE significantly increased it by an additional $4 \%(\Delta F=13.04, p<0.001)$. In the alternative model, NMRE and control variables explained a $16 \%$ variance in social anxiety symptoms and adding four IER strategies significantly incremented it for an additional $5 \%(\Delta F=4.27, p=0.002)$. We also tested the alternative model in a single path analysis (see Appendix A) and examined the indirect relationships between NMRE and social anxiety symptoms through IER strategies. The 
only significant relationship was the one between NMRE and social anxiety symptoms through soothing, coefficient $=-0.05, S E=0.03,95 \%$ CI $[-0.14,-0.01]$.

Lastly, the DDA analysis was conducted, and the results were reported in Appendix B Briefly, the DDA results generally supported IER $\rightarrow$ NMRE direction. None of the tested properties indicated an NMRE $\rightarrow$ IER relationship, while the distributional characteristics comparisons of predictors and residuals provided at least partial support for the IER $\rightarrow$ NMRE relationship for enhancing positive affect, soothing, and social modeling. The independence comparisons favored the IER $\rightarrow$ NMRE relationship for enhancing positive affect.

\section{Discussion}

The main goal of the current study was to investigate the relationship between IER and social anxiety symptoms, and for this purpose, two studies were reported. The first study showed that IER strategies, such as perspective-taking, soothing, and social modeling, are significant correlates of social anxiety symptoms after controlling depression, suppression and reappraisal. These findings were replicated in Study 2, which was conducted with a community sample. Moreover, we found that NMRE mediated the relationship between two of the IER strategies (i.e. enhancing positive affect and soothing) and social anxiety symptoms.

The first important finding is that three of the four IER strategies were significantly related to social anxiety symptoms, accounting for depression, suppression and reappraisal. Specifically, perspective-taking was negatively related to social anxiety symptoms, while soothing and social modeling were positively related to social anxiety symptoms. Overall, these results are partly consistent with limited studies, which suggest that IER and social anxiety are associated. Consistent with our findings, Hofmann et al. (2016) and Koç et al. (2019) reported that social anxiety symptoms are positively linked to soothing and social modeling. However, contrary to the positive association between perspective-taking and social anxiety reported in both studies, we found that perspective-taking is a negative correlate of social anxiety.

Soothing refers to seeking out other people to regulate emotions (Hofmann et al., 2016), and our study indicated that this strategy is related to social anxiety symptoms. We offer two explanations regarding how soothing can be associated with social anxiety. First, constantly seeking others to regulate negative emotions can undermine one's belief in own emotion regulation skills. Thus, overusing soothing strategy diminishes the confidence one has in their emotion regulation skills. Cognitive-behavioural models of SAD (e.g. Clark \& Wells, 1995; Hofmann, 2007) suggest that negative core beliefs or negative self-perceptions have an important role in the aetiology and maintenance of social anxiety. Moreover, numerous studies supported the argument that negative beliefs about self are an essential part of SAD (Wong et al., 2017; Boden et al., 2012; also see Macarthur, 2013). Thus, coping with negative emotions through others may contribute to the maintenance of one's negative beliefs and, consequently, lead social anxiety. Secondly, soothing can be conceptualised as a safety behaviour (Hofmann, 2014). Especially, in social settings, being with other people (e.g. friends, family members) may help regulate negative emotions caused by a social situation. However, this strategy can contribute to social anxiety symptoms in the long run. Several studies showed that safety behaviours were associated with social anxiety (Kocovski et al., 2015; McManus et al., 2008). Overall, soothing can be a problematic strategy to cope with negative emotions and may result in an increase in social anxiety symptoms.

Our results indicated that social modeling—-described as one's attempt to regulate emotions by observing how other people deal with such situations (Sarısoy-Aksüt \& Gençöz, 2020) is another significant correlate of social anxiety symptoms. This finding is consistent with previous studies that found a positive correlation between social modeling and social anxiety (Hofmann et al., 2016; Koç et al., 2019). One explanation for this result may be that, because individuals with high social anxiety experience more negative emotions (Farmer \& Kashdan, 2012), they more often use this strategy. Moreover, low confidence in one's emotion regulation skills and negative beliefs about self encourage one to observe others. In support of this view, in his comprehensive model, Hofmann (2007) suggests that low perceived emotional control is an important maintaining factor in SAD. However, further research is needed to understand why social modeling is positively associated with social anxiety.

Perspective-taking pertains to using other people to view the situations more favourably and be reminded not to worry (Gökdağ, 2021; Hofmann et al., 2016). Contrary to other IER strategies (soothing and social modeling), perspective-taking was negatively associated with social anxiety symptoms in the current study. This result suggests that perspective-taking may be an adaptive strategy in regulating negative emotions in the context of social anxiety. We believe one reason perspective-taking has a negative association with social anxiety symptoms may be related to its cognitive restructuring aspects. Cognitive restructuring refers to the modification of maladaptive appraisals and beliefs that maintain psychological disturbance (Clark, 2014), which is an essential part of the cognitive-behavioural therapy of SAD (Heimberg, 2002; Hope et al., 2010; Pelissolo et al., 2019). Consulting other people when one experiences negative emotions can help change thoughts about stressful situations, which in turn may reduce negative emotions, such as 
anxiety. In the context of social anxiety, others may encourage individuals with high social anxiety to re-examine the social situation that causes anxiety. Thus, core features of SAD, such as overestimation of the probability of social mishaps and social cost of these mishaps (Hofmann \& Otto, 2017) can be modified. Thereby, individuals with high social anxiety view the social situations more positively and less threatening, which in turn may contribute to the alleviation of social anxiety symptoms.

It is important to note that the zero-order correlation between perspective-taking and social anxiety symptoms was not significant, while the relationship in the regression was negative, which may indicate negative suppression. A negative suppression is described as a situation in which there is a positive correlation between two predictor variables and the correlation of one with the criterion variable is weaker compared to the correlation of the other (Massen \& Bakker 2001). Given the low variance inflation factor scores, the fact that exclusion of control variables did not affect the pattern of results and the theoretical plausibility, we interpreted the negative relationship between perspectivetaking and social anxiety. However, the negative effect of perspective-taking exists with the complex relationships of other correlates, which should be taken into account in the interpretation of the relationship.

The second finding of the study was the mediating role of NMRE in the relationship between IER and social anxiety symptoms. It was found that NMRE mediate the relationship between two IER strategies (i.e. enhancing positive affect and soothing) and social anxiety symptoms. Enhancing positive affect refers to seeking out other people to share and increase positive emotions, such as happiness and joy (Hofmann et. al., 2016). Although enhancing positive affect was not directly related to social anxiety symptoms, the indirect relationship between enhancing positive affect and social anxiety through NMRE was significant. The results of studies examining enhancing positive affect and its relationships with various psychological disturbances indicated that it may not be a maladaptive strategy (Ray-Yol et al., 2020). For example, several studies found that enhancing positive affect is negatively associated with depression (Altan-Atalay \& Saritas-Atalar, 2019; Koç et al., 2019), and others found no correlation between enhancing positive affect and various psychological problems, such as depression and anxiety (Gökda ̆ , 2021; Gökdağ \& Naldöken, 2021; Hofmann et al., 2016). In the context of social anxiety, limited studies report no association between enhancing positive affect and social anxiety (Hofmann et al., 2016; Koç et al., 2019). Contrary to other dimensions of IER, enhancing positive affect describes attempts to enhance emotions when one is in a positive mood. Experiencing positive emotions and sharing them with others may strengthen one's beliefs regarding emotion regulation skills, making social situations more manageable.
Thus, sharing positive affect with others may bring about more adaptive beliefs of both self and social settings, which in turn decrease social anxiety symptoms.

The study also showed that the relationship between soothing and social anxiety symptoms was mediated by NMRE. Accordingly, soothing was related to NMRE negatively, and in turn, NMRE were negatively associated with social anxiety symptoms. Negative self-beliefs are an important part of SAD (Clark \& Wells, 1995; Hofmann, 2007), and approaching other people to cope with negative emotions might undermine one's own emotion regulation abilities. Regulating emotions through others may contribute to the maintenance of negative self-beliefs about one's emotion regulation ability, which in turn increase social anxiety symptoms.

Even though we hypothesized that NMRE mediate the relationship between all four IER strategies and social anxiety, the indirect relationships were significant for enhancing positive affect and soothing. The indirect associations of perspective-taking and social modeling with social anxiety through NMRE were not significant. A similar pattern of results was documented in a study conducted by RayYol and Altan-Atalay (2020), which reported that NMRE did not significantly mediate the relationship between IER strategies and psychological distress except for soothing. Ray-Yol and Altan-Atalay (2020) argued that both perspective-taking and social modeling may potentially provide individuals an opportunity to acquire emotion regulation skills. In other words, when one experiences a stressful situation, approaching others to get their opinion (perspective taking) and observing others to learn how they cope with negative feelings (social modeling) may not undermine one's expectancies of own coping skills. Therefore, unlike soothing, perspective-taking and social modeling may not be associated with lower NMRE.

Although not directly related to the study findings, it is important to note that about half of the participants in Study 1 scored above the clinical range of LSAS. We believe three factors may explain the high percentage of individuals that scored above the clinical range. First, because the sample was predominantly female $(86.3 \%)$ and social anxiety disorder is more common among women (Asher \& Aderka, 2018; Asher et al., 2017; Caballo et al., 2014), LSAS scores in our study may be relatively high. Similarly, participant age may be another factor for high LSAS scores. Our sample consists of mostly young people (mean age $=20.28$ years) and several studies reported high rates of social anxiety among young people (Jefferies \& Ungar, 2020; Magee et al., 1996). Lastly, the data was collected during the COVID-19 pandemic and some studies reported that it is associated with elevated social anxiety (Hawes et al., 2021). Nevertheless, the results of participants who scored high on LSAS were mostly similar to 
the whole sample, suggesting that the unusually high rates of social anxiety symptoms in the sample did not have a considerable influence on our findings.

Although the current study offers important findings regarding the IER-social anxiety symptoms relationship, it has several limitations. First, this study introduced NMRE as an intermediary process between IER and social anxiety based on the argument that frequently resorting to interpersonal forms of emotion regulation may hinder one's expectancies about their emotion regulation abilities, which in turn contributes to the maintenance of social anxiety symptoms. However, competing models with an alternative ordering of relationships among IER, NMRE and social anxiety symptoms can be plausible and cannot be ruled out with the crosssectional and non-experimental design utilized in the current study. One such model where the IER strategies mediated the relationship between NMRE and social anxiety symptoms was tested and compared against our hypothesised model. The results showed that they account for a similar amount of variance in social anxiety symptoms. In the alternative model, one indirect relationship was significant. We further scrutinized the ordering of the relationship between IER and NMRE by employing DDA. The results were more supportive of NMRE $\rightarrow$ IER direction. Another alternative model where social anxiety leads to the use of IER strategies would also be plausible. Past research documented that social anxiety is related to dysfunctional emotion regulation strategies (Farmer \& Kashdan, 2012; Kocovski et al., 2005). Thus, it can be argued that people with social anxiety may be more inclined to seek others for comfort or be more observative of others to learn how to cope. However, even though the accumulated research that linked NMRE to emotion regulation strategies has urged us to compare the ordering of the relationship between IER and NMRE, we refrain from testing additional alternative models for an important reason. Given the cross-sectional design of our study, it is not possible to decide on the correct or more plausible model specification by comparing the significance of parameter estimates or the number of significant mediated relationships of the alternative ordering of variables (Wiedermann \& von Eye, 2015). Such practices do not provide strong evidence on a plausible model and can be misleading (Lemmer \& Gollwitzer, 2017; Thoemmes, 2015). Heeding these suggestions, we present the alternative model for informative purposes and abstain from making conclusive statements about model plausibility. Moreover, we regard the findings of the current research as preliminary evidence which should be subjected to further examination and invite future studies to investigate the causal relationship between IER, NMRE and social anxiety symptoms by utilising designs that let modelling feedback loops and bidirectional relationships between variables.

Second, the participants were recruited by employing convenience sampling. This is particularly an issue for Study 2 data, which was collected from a small social media circle. Such a sampling procedure may bring about two points that should be noted. First, the sample recruited with the sampling method used in Study 2 may have distinct participant characteristics, which would result in biased results unrepresentative of the population. Even though the pattern of the relationship between IER and social anxiety symptoms was similar across Study 1 and Study 2 samples, it remains a concern. Second, related to the first point, the sampling method of Study 2 limits the generalizability of the results. Data collected from social media do not capture the whole population and have skewed demographics (Davis \& Love, 2019). This may become a more prominent issue when, as in Study 2, the data was collected from a smaller social circle. Thus, the results should be interpreted with this limitation. Future studies that utilise random sampling procedures are warranted. Third, both studies were conducted with nonclinical participants. Studies utilising clinical samples would help generalise findings. Fourth the participants in Study 1 were disproportionately women. Even though Study 2 confirmed the findings of Study 1 in a sample with more even sex distribution, caution is warranted in generalising Study 1 results. Lastly, although the studies showed the relationship between IER and social anxiety symptoms after controlling for suppression and reappraisal, these two constitute a small subset of intrapersonal emotion regulation strategies. Further studies can include other strategies, such as avoidance, acceptance, and rumination (Kraiss et al., 2020) to investigate the role of IER in social anxiety symptoms, accounting for such strategies.

\section{Conclusion}

Notwithstanding the limitations, the present study has important contributions to emerging research on IER. To the best of our knowledge, this is the first study to examine the role of IER on social anxiety symptoms in both student and community samples. Our results indicated that, while IER strategies, such as soothing and social modeling, might play a detrimental role in social anxiety symptoms, perspectivetaking can be a functional strategy. Moreover, NMRE has a substantial role in the relationship between IER and social anxiety symptoms. Considering IER strategies and beliefs about emotion regulation skills may be helpful in the treatment of SAD.

\section{Appendix A}

(See Fig. 2). 
Fig. 2 Path analysis results of alternative model

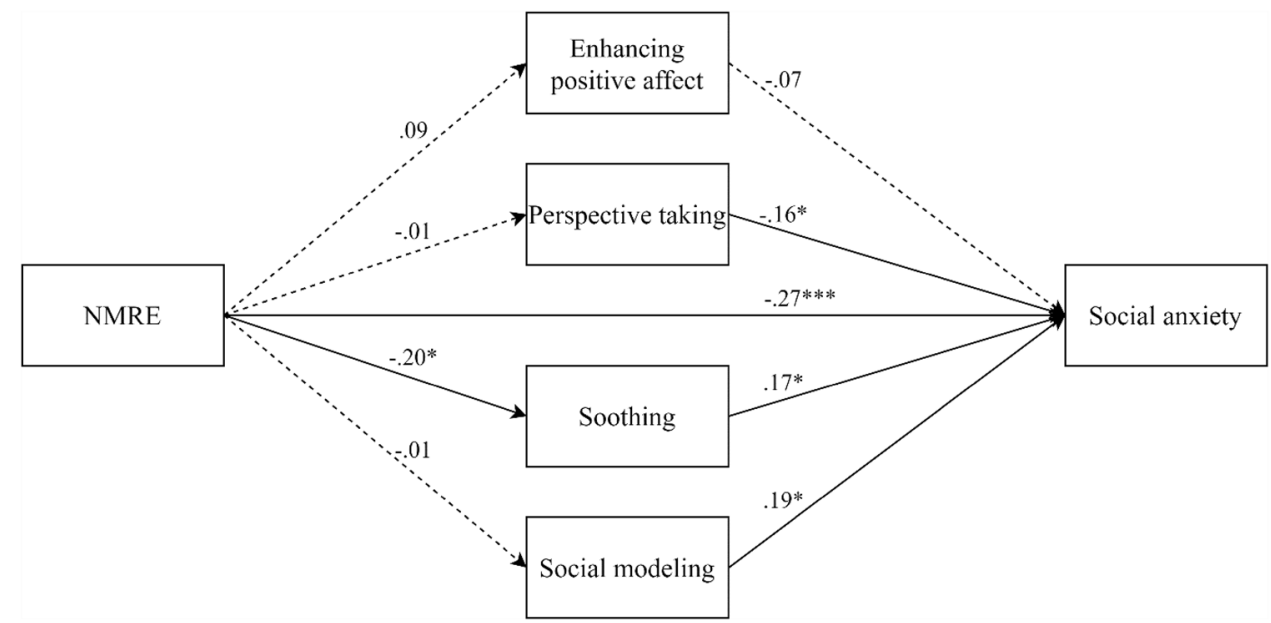

Note. $N=271$. Standardized estimates were reported. The dashed lines represent nonsignificant paths. The controls were estimated but were not displayed for brevity. NMRE: negative mood regulation expectancies.

$* p<.05, * * p<.01, * * * p<.001$.

\section{Appendix B}

We utilized DDA to test the direction of the relationship between four IER strategies and NMRE by using the SPSS macro provided by Wiedermann and Li (2021). Since our focus was specifically on the causal order of the relationship between the two constructs, we investigated the relationships in isolation. We report the results of four analyses, which denote the direction comparisons of the relationship between NMRE and each IER strategy. Since we hypothesised that IER precedes NMRE, the target model denotes IER $\rightarrow$ NMRE while the alternative model denotes $\mathrm{NMRE} \rightarrow$ IER in the following tests.

The skewness $(p=0.66)$ and excess-kurtosis $(p=0.16)$ of NMRE were not significantly different from zero. Of the four IER strategies, the skewness $(p<0.001)$ and excess kurtosis $(p=0.02)$ of enhancing positive affect, the excess kurtosis of soothing $(p=0.002)$, and the skewness $(p=0.05)$ and excess kurtosis $(p=0.04)$ of social modeling were significantly different from zero. Moreover, the bootstrap tests with 1000 resamples showed that the confidence intervals did not include zero for the skewness and excess kurtosis difference between NMRE and enhancing positive affect, which indicates a significant difference between the distributional properties of the two variables. Altogether, the comparisons suggest at least partial support for the IER $\rightarrow$ NMRE relationship except for perspective-taking considering the first DDA component.

Second, we examined the residual distribution of competing models. The results were shown in Table 1. Results indicated that none of the distributional properties of target model residuals significantly deviate from zero. On the other hand, alternative model residuals were significantly different from zero for at least for one distributional property for enhancing positive affect, soothing, and social modeling. Moreover, the bootstrap analysis with 1000 resamples showed that the skewness difference between competing models of enhancing positive affect-NMRE relationship was significantly different. Taken together, the results suggest the IER $\rightarrow$ NMRE relationship except for the perspectivetaking-NMRE relationship (see Table 5).

Lastly, we examined the independence properties of predictors and residuals of the competing models. We investigated the results of the Breusch-Pagan homoscedasticity test (BP test) and Hilbert-Schmidt independence criterion (HSIC). Moreover, we examined different combinations of nonlinear correlations between predictors and residuals. Results supported enhancing positive affect $\rightarrow$ NMRE direction as indicated by non-significant target model, $\chi^{2}(1)=1.77, p=0.62$, HSIC $=0.08, p=0.11$, and significant alternative model, $\chi^{2}(1)=12.73, p=0.005$, HSIC $=0.23$, $p=0.005$. Moreover, the three non-linear correlation combinations were not significant for the target model, while two of them were significant for the alternative model. The BP test and HSIC results for the perspective-takingNMRE relationship were not significant for the target model $\chi^{2}(1)=1.10, p=0.30$, HSIC $=0.09, p=0.16$, and the alternative model $\chi^{2}(1)=2.88, p=0.09$, HSIC $=0.09, p=0.13$. The three non-linear correlation combinations were not significant for the target model, while one of them was significant 
Table 5 The comparison of distributional properties of model residuals

\begin{tabular}{|c|c|c|c|c|c|c|c|c|}
\hline \multirow[t]{3}{*}{ Variable } & \multicolumn{4}{|c|}{ Target model $^{\mathrm{a}}$} & \multicolumn{4}{|c|}{ Alternative model $^{\mathrm{b}}$} \\
\hline & \multicolumn{2}{|c|}{$\begin{array}{l}\text { Skewness } \\
\text { NMRE }\end{array}$} & \multicolumn{2}{|c|}{$\begin{array}{l}\text { Ex-kurtosis } \\
\text { NMRE }\end{array}$} & \multicolumn{2}{|l|}{ Skewness } & \multicolumn{2}{|c|}{ Ex-kurtosis } \\
\hline & $b$ & $z$ & $b$ & $z$ & $b$ & $z$ & $b$ & $z$ \\
\hline Enhancing positive affect & 0.003 & 0.02 & -0.09 & -0.11 & $-0.96 * * *$ & -5.68 & $0.91 *$ & 2.47 \\
\hline Perspective-taking & 0.08 & 0.53 & -0.41 & -1.57 & 0.26 & 1.76 & -0.45 & -1.78 \\
\hline Soothing & 0.01 & 0.10 & -0.46 & -1.86 & -0.005 & -0.04 & $-0.64 * *$ & -3.05 \\
\hline Social modeling & 0.11 & 0.75 & -0.40 & -1.50 & $-0.31 *$ & -2.11 & -0.42 & -1.63 \\
\hline
\end{tabular}

${ }^{\text {a }}$ Target models describe the IER $\rightarrow$ NMRE relationship in which the skewness and kurtosis values denote the distributional properties of IER $\rightarrow$ NMRE residuals

${ }^{b}$ Alternative models describe the NMRE $\rightarrow$ IER relationship in which the skewness and kurtosis values denote the distributional properties of NMRE $\rightarrow$ IER residuals

Ex-kurtosis excess kurtosis

$* p<0.05, * * p<0.01, * * * p<0.001$ for the alternative model. For the relationship between soothing and NMRE, the BP test and HSIC indicated that both target model, $\chi^{2}(1)=1.97, p=0.16$, HSIC $=0.10$, $p=0.14$, and alternative model $\chi^{2}(1)=0.003, p=0.95$, HSIC $=0.07, p=0.21$, were not significant. Moreover, none of the non-linear correlation combinations were significant. The BP test and HSIC results did not indicate dependence in target model, $\chi^{2}(1)=2.45, p=0.12$, HSIC $=0.10, p=0.07$, and alternative model $\chi^{2}(1)=0.37, p=0.55$, HSIC $=0.10$, $p=0.06$ of social modelling-NMRE relationship. None of the non-linear correlation combinations were significant.

Acknowledgements We are grateful to Ege University Planning and Monitoring Coordination of Organizational Development and Directorate of Library and Documentation for their support in editing and proofreading service of this study.

Funding This research did not receive any specific grant from funding agencies in the public, commercial, or not-for-profit sectors.

\section{Declarations}

Conflict of Interest Koray Akkuş and Mehmet Peker declare that they have no conflict of interest.

Ethical Approval All procedures performed in the two studies involving human participants were in accordance with the ethical standards of the institutional and/or national research committee and with the 1964 Helsinki declaration and its later amendments or comparable ethical standards.

\section{References}

Acarturk, C., de Graaf, R., van Straten, A., Have, M., \& Cuijpers, P. (2008). Social phobia and number of social fears, and their association with comorbidity, health-related quality of life and help seeking. Social Psychiatry and Psychiatric Epidemiology, 43(4), 273-279. https://doi.org/10.1007/s00127-008-0309-1

Altan-Atalay, A., \& Saritas-Atalar, D. (2019). Interpersonal emotion regulation strategies: How do they interact with negative mood regulation expectancies in explaining anxiety and depression? Current Psychology. https://doi.org/10.1007/s12144-019-00586-2

American Psychiatric Association. (2013). Diagnostic and statistical manual of mental disorders (5th ed.). American Psychiatric Publishing.

Asher, M., \& Aderka, I. M. (2018). Gender differences in social anxiety disorder. Journal of Clinical Psychology, 74(10), 1730-1741. https://doi.org/10.1002/jclp.22624

Asher, M., Asnaani, A., \& Aderka, I. M. (2017). Gender differences in social anxiety disorder: A review. Clinical Psychology Review, 56, 1-12. https://doi.org/10.1016/j.cpr.2017.05.004

Aydemir, Ö., Güvenir, T., Küey, L., \& Kültür, S. (1997). Validity and reliability of Turkish version of hospital anxiety and depression scale. Turkish Journal of Psychiatry, 8(4), 280-287.

Beesdo, K., Bittner, A., Pine, D. S., Stein, M. B., Höfler, M., Lieb, R., \& Wittchen, H.-U. (2007). Incidence of social anxiety disorder and the consistent risk for secondary depression in the first three decades of life. Archives of General Psychiatry, 64(8), 903-912. https://doi.org/10.1001/archpsyc.64.8.903

Bjelland, I., Dahl, A. A., Haug, T. T., \& Neckelmann, D. (2002). The validity of the hospital anxiety and depression scale: An updated literature review. Journal of Psychosomatic Research, 52(2), 69-77. https://doi.org/10.1016/S0022-3999(01)00296-3

Boden, M. T., John, O. P., Goldin, P. R., Werner, K., Heimberg, R. G., \& Gross, J. J. (2012). The role of maladaptive beliefs in cognitive-behavioral therapy: Evidence from social anxiety disorder. Behaviour Research and Therapy, 50(5), 287-291. https://doi.org/10.1016/j.brat.2012.02.007

Caballo, V. E., Salazar, I. C., Irurtia, M. J., Arias, B., Hofmann, S. G., \& Ciso-A Research Team. (2014). Differences in social anxiety between men and women across 18 countries. Personality and Individual Differences, 64, 35-40. https://doi.org/10. 1016/j.paid.2014.02.013

Catanzaro, S. J., Backenstrass, M., Miller, S. A., Mearns, J., Pfeiffer, N., \& Brendalen, S. (2014). Prediction of symptoms of emotional distress by mood regulation expectancies and affective traits. International Journal of Psychology, 49(6), 471-479. https://doi.org/10.1002/ijop.12062

Catanzaro, S. J., \& Greenwood, G. (1994). Expectancies for negative mood regulation, coping, and dysphoria among college students. 
Journal of Counseling Psychology, 41(1), 34-44. https://doi. org/10.1037/0022-0167.41.1.34

Catanzaro, S. J., \& Mearns, J. (1990). Measuring generalized expectancies for negative mood regulation: Initial scale development and implications. Journal of Personality Assessment, 54(3-4), 546-563. https://doi.org/10.1080/00223891.1990.9674019

Catanzaro, S. J., \& Mearns, J. (1999). Mood-related expectancy, emotional experience, and coping behavior. In I. Kirsch (Ed.), How expectancies shape experience (pp. 67-91). American Psychological Association.

Catanzaro, S. J., Wasch, H. H., Kirsch, I., \& Mearns, J. (2000). Coping-related expectancies and dispositions as prospective predictors of coping responses and symptoms. Journal of Personality, 68(4), 757-788. https://doi.org/10.1111/1467-6494.00115

Clark, D. A. (2014). Cognitive restructuring. In S. G. Hofmann (Ed.), The Wiley handbook of cognitive behavioral therapy (pp. 1-22). Wiley-Blackwell.

Clark, D. A., \& Beck, A. T. (2010). Cognitive therapy of anxiety disorders: Science and practice. Guilford Press.

Clark, D. M., \& Wells, A. (1995). A cognitive model of social phobia. In R. G. Heimberg \& M. R. Liebowitz (Eds.), Social phobia: Diagnosis, assessment, and treatment (pp. 69-93). Guilford Press.

Cloitre, M., Chase Stovall-McClough, K., Miranda, R., \& Chemtob, C. M. (2004). Therapeutic alliance, negative mood regulation, and treatment outcome in child abuse-related posttraumatic stress disorder. Journal of Consulting and Clinical Psychology, 72(3), 411-416. https://doi.org/10.1037/0022-006X.72.3.411

Cloitre, M., Stovall-McClough, K. C., Nooner, K., Zorbas, P., Cherry, S., Jackson, C. L., Gan, W., \& Petkova, E. (2010). Treatment for PTSD related to childhood abuse: A randomized controlled trial. American Journal of Psychiatry, 167(8), 915-924. https://doi.org/10.1176/appi.ajp.2010.09081247

Crome, E., Grove, R., Baillie, A. J., Sunderland, M., Teesson, M., \& Slade, T. (2015). DSM-IV and DSM-5 social anxiety disorder in the Australian community. Australian \& New Zealand Journal of Psychiatry, 49(3), 227-235. https://doi.org/10.1177/00048 67414546699

Davis, J. L., \& Love, T. P. (2019). Generalizing from social media data: A formal theory approach. Information, Communication \& Society, 22(5), 637-647.

Davis, R. N., Andresen, E. N., Trosko, M., Massman, P. J., \& Lovejoy, M. C. (2005). Negative mood regulation (NMR) expectancies: A test of incremental validity. Personality and Individual Differences, 39(2), 263-270. https://doi.org/10.1016/j.paid. 2005.01.002

Dryman, M. T., \& Heimberg, R. G. (2018). Emotion regulation in social anxiety and depression: A systematic review of expressive suppression and cognitive reappraisal. Clinical Psychology Review, 65, 17-42. https://doi.org/10.1016/j.cpr.2018.07.004

Farmer, A. S., \& Kashdan, T. B. (2012). Social anxiety and emotion regulation in daily life: Spillover effects on positive and negative social events. Cognitive Behaviour Therapy, 41(2), 152-162. https://doi.org/10.1080/16506073.2012.666561

Gökdağ, C. (2021). How does interpersonal emotion regulation explain psychological distress? The roles of attachment style and social support. Personality and Individual Differences, 176, 110763. https://doi.org/10.1016/j.paid.2021.110763

Gökdağ, C., \& Naldöken, B. (2021). The relationship between interpersonal emotion regulation, personality traits, and psychopathology symptoms. Turkish Journal of Clinical Psychiatry, 24(1), 41-52. https://doi.org/10.5505/kpd.2020.79106

Gökda ̆, C., Sorias, O., Kiran, S., \& Ger, S. (2019). Adaptation of the interpersonal emotion regulation questionnaire to the Turkish language and investigation of its psychometric properties.
Turkish Journal of Psychiatry, 30(1), 57-66. https://doi.org/10. 5080/u23067

Gross, J. J., \& John, O. P. (2003). Individual differences in two emotion regulation processes: Implications for affect, relationships, and well-being. Journal of Personality and Social Psychology, 85(2), 348-362. https://doi.org/10.1037/0022-3514.85.2.348

Hawes, M. T., Szenczy, A. K., Klein, D. N., Hajcak, G., \& Nelson, B. D. (2021). Increases in depression and anxiety symptoms in adolescents and young adults during the COVID-19 pandemic. Psychological Medicine. https://doi.org/10.1017/S003329172 0005358

Heimberg, R. G. (2002). Cognitive-behavioral therapy for social anxiety disorder: Current status and future directions. Biological Psychiatry, 51(1), 101-108. https://doi.org/10.1016/s0006-3223(01) 01183-0

Heimberg, R. G., Brozovich, F. A., \& Rapee, R. M. (2010). A Cognitive behavioral model of social anxiety disorder. Social Anxiety. https://doi.org/10.1016/b978-0-12-375096-9.00015-8

Heimberg, R. G., Brozovich, F. A., \& Rapee, R. M. (2014). A cognitive-behavioral model of social anxiety disorder. Social Anxiety. https://doi.org/10.1016/b978-0-12-394427-6.00024-8

Heimberg, R. G., Horner, K. J., Juster, H. R., Safren, S. A., Brown, E. J., Schneier, F. R., \& Liebowitz, M. R. (1999). Psychometric properties of the Liebowitz social anxiety scale. Psychological Medicine, 29(1), 199-212. https://doi.org/10.1017/s003329179 8007879

Hofmann, S. G. (2007). Cognitive factors that maintain social anxiety disorder: A comprehensive model and its treatment implications. Cognitive Behaviour Therapy, 36(4), 193-209. https://doi. org/10.1080/16506070701421313

Hofmann, S. G. (2014). Interpersonal emotion regulation model of mood and anxiety disorders. Cognitive Therapy and Research, 38(5), 483-492. https://doi.org/10.1007/s10608-014-9620-1

Hofmann, S. G., Carpenter, J. K., \& Curtiss, J. (2016). Interpersonal emotion regulation questionnaire (IERQ): Scale development and psychometric characteristics. Cognitive Therapy and Research, 40(3), 341-356. https://doi.org/10.1007/ s10608-016-9756-2

Hofmann, S. G., \& Otto, M. W. (2017). Cognitive behavioral therapy for social anxiety disorder: Evidence-based and disorder specific treatment techniques. Routledge.

Hope, D. A., Heimberg, R. G., \& Turk, C. L. (2010). Managing social anxiety: A cognitive-behavioral therapy approach (2nd ed.). Oxford University Press.

Jazaieri, H., Morrison, A. S., Goldin, P. R., \& Gross, J. J. (2015). The role of emotion and emotion regulation in social anxiety disorder. Current Psychiatry Reports, 17(1), 531. https://doi.org/10.1007/ s11920-014-0531-3

Jefferies, P., \& Ungar, M. (2020). Social anxiety in young people: A prevalence study in seven countries. PLoS ONE, 15(9), e0239133. https://doi.org/10.1371/journal.pone.0239133

Kassel, J. D., Bornovalova, M., \& Mehta, N. (2007). Generalized expectancies for negative mood regulation predict change in anxiety and depression among college students. Behaviour Research and Therapy, 45(5), 939-950. https://doi.org/10.1016/j.brat.2006. 07.014

Kaymakçığlu, Ç. (2001). Effects of perceived stress and social support, expectancies and coping style on depression and anxiety. [Unpublished master's thesis]. Boğaziçi University

Kessler, R. C., Berglund, P., Demler, O., Jin, R., Merikangas, K. R., \& Walters, E. E. (2005). Lifetime prevalence and age-of-onset distributions of DSM-IV disorders in the national comorbidity survey replication. Archives of General Psychiatry, 62(6), 593-602. https://doi.org/10.1001/archpsyc.62.6.593

Koç, M. S., Aka, B. T., Doğruyol, B., Curtiss, J., Carpenter, J. K., \& Hofmann, S. G. (2019). Psychometric properties of the Turkish 
version of the interpersonal emotion regulation questionnaire (IERQ). Journal of Psychopathology and Behavioral Assessment, 41(2), 294-303. https://doi.org/10.1007/s10862-019-09732-3

Kocovski, N. L., Endler, N. S., Rector, N. A., \& Flett, G. L. (2005). Ruminative coping and post-event processing in social anxiety. Behaviour Research and Therapy, 43(8), 971-984. https://doi.org/ 10.1016/j.brat.2004.06.015

Kocovski, N. L., MacKenzie, M. B., Albiani, J. J., Battista, S. R., Noel, S., Fleming, J. E., \& Antony, M. M. (2015). Safety behaviors and social anxiety: An examination of the social phobia safety behaviours scale. Journal of Psychopathology and Behavioral Assessment, 38(1), 87-100. https://doi.org/10.1007/s10862-015-9498-6

Koyuncu, A., İnce, E., Ertekin, E., \& Tükel, R. (2019). Comorbidity in social anxiety disorder: Diagnostic and therapeutic challenges. Drugs in Context, 8, 1-13. https://doi.org/10.7573/dic.212573

Kraiss, J. T., Peter, M., Moskowitz, J. T., \& Bohlmeijer, E. T. (2020). The relationship between emotion regulation and well-being in patients with mental disorders: A meta-analysis. Comprehensive Psychiatry, 102, 152189. https://doi.org/10.1016/j.comppsych. 2020.152189

Ledley, D. R., Huppert, J. D., Foa, E. B., Davidson, J. R., Keefe, F. J., \& Potts, N. L. (2005). Impact of depressive symptoms on the treatment of generalized social anxiety disorder. Depression and Anxiety, 22(4), 161-167. https://doi.org/10.1002/da.20121

Lemmer, G., \& Gollwitzer, M. (2017). The "true" indirect effect won't (always) stand up: When and why reverse mediation testing fails. Journal of Experimental Social Psychology, 69, 144-149. https:// doi.org/10.1016/j.jesp.2016.05.002

Liebowitz, M. R. (1987). Social phobia. Modern Problems in Pharmacopsychiatry, 22, 141-173. https://doi.org/10.1159/000414022

Macarthur, J. (2013). An integrative approach to addressing core beliefs in social anxiety. Journal of Psychotherapy Integration, 23(4), 386-396. https://doi.org/10.1037/a0035043

Magee, W. J., Eaton, W. W., Wittchen, H. U., McGonagle, K. A., \& Kessler, R. C. (1996). Agoraphobia, simple phobia, and social phobia in the national comorbidity survey. Archives of General Psychiatry, 53(2), 159-168. https://doi.org/10.1001/archpsyc. 1996.01830020077009

Massen, G. H., \& Bakker, A. B. (2001). Suppressor variables in path models. Sociological Methods \& Research, 30(2), 241-270. https://doi.org/10.1177/0049124101030002004

Mazur-Socha, Z., \& Przepiórka, A. (2021a). A systematic review of correlates of negative mood regulation expectancies. Personality and Individual Differences, 179, 110930. https://doi.org/10. 1016/j.paid.2021.110930

Mazur-Socha, Z., \& Przepiórka, A. (2021b). Affective correlates of negative mood regulation expectancies: A systematic review and meta-analysis. Journal of Research in Personality. https://doi.org/ 10.1016/j.jrp.2021.104098

McManus, F., Sacadura, C., \& Clark, D. M. (2008). Why social anxiety persists: An experimental investigation of the role of safety behaviours as a maintaining factor. Journal of Behavior Therapy and Experimental Psychiatry, 39(2), 147-161. https://doi.org/10. 1016/j.jbtep.2006.12.002

Mrnak-Meyer, J., Tate, S. R., Tripp, J. C., Worley, M. J., Jajodia, A., \& Mcquaid, J. R. (2011). Predictors of suicide-related hospitalization among U.S. veterans receiving treatment for comorbid depression and substance dependence. Suicide and Life-Threatening Behavior, 41(5), 532-542. https://doi.org/10.1111/j.1943278x.2011.00051.x

Muthén, L. K., \& Muthén, B. O. (2017). Mplus user's guide (8th ed.). Muthén \& Muthén.

O’Toole, M. S., Hougaard, E., \& Mennin, D. S. (2013). Social anxiety and emotion knowledge: A meta-analysis. Journal of Anxiety Disorders, 27(1), 98-108. https://doi.org/10.1016/j.janxdis.2012. 09.005
Pelissolo, A., AbouKassm, S., \& Delhay, L. (2019). Therapeutic strategies for social anxiety disorder: Where are we now? Expert Review of Neurotherapeutics, 19(12), 1179-1189. https://doi.org/ $10.1080 / 14737175.2019 .1666713$

Rapee, R. M., \& Heimberg, R. G. (1997). A cognitive-behavioral model of anxiety in social phobia. Behaviour Research and Therapy, 35(8), 741-756. https://doi.org/10.1016/S0005-7967(97)00022-3

Ray-Yol, E., \& Altan-Atalay, A. (2020). Interpersonal emotion regulation and psychological distress: What is the function of negative mood regulation expectancies in this relationship? Psychological Reports. https://doi.org/10.1177/0033294120968086

Ray-Yol, E., Ülbe, S., Temel, M., \& Altan-Atalay, A. (2020). Interpersonal emotion regulation strategies: Can they function differently under certain conditions? Current Psychology. https:// doi.org/10.1007/s12144-020-00771-8

Sarısoy-Aksüt, G., \& Gençöz, T. (2020). Psychometric properties of the interpersonal emotion regulation questionnaire (IERQ) in Turkish samples. Current Psychology. https://doi.org/10.1007/ s12144-019-00578-2

Soykan, Ç., Özgüven, H. D., \& Gençöz, T. (2003). Liebowitz social anxiety scale: The Turkish version. Psychological Reports, 93(3), 1059-1069. https://doi.org/10.2466/pr0.2003.93.3f.1059

Spokas, M., Luterek, J. A., \& Heimberg, R. G. (2009). Social anxiety and emotional suppression: The mediating role of beliefs. Journal of Behavior Therapy and Experimental Psychiatry, 40(2), 283-291. https://doi.org/10.1016/j.jbtep.2008.12.004

Stangier, U. (2016). New developments in cognitive-behavioral therapy for social anxiety disorder. Current Psychiatry Reports, 18(3), 25. https://doi.org/10.1007/s11920-016-0667-4

Stein, D. J., Lim, C. C. W., Roest, A. M., de Jonge, P., Aguilar-Gaxiola, S., Al-Hamzawi, A., Alonso, J., Benjet, C., Bromet, E. J., Bruffaerts, R., de Girolamo, G., Florescu, S., Gureje, O., Haro, J. M., Harris, M. G., He, Y., Hinkov, H., Horiguchi, I., Hu, C., ... WHO World Mental Health Survey Collaborators. (2017). The cross-national epidemiology of social anxiety disorder: Data from the world mental health survey initiative. BMC Medicine, 15(1), 1-21. https://doi.org/10.1186/s12916-017-0889-2

Stein, M. B., \& Stein, D. J. (2008). Social anxiety disorder. The Lancet, 371(9618), 1115-1125. https://doi.org/10.1016/s01406736(08)60488-2

Sung, S. C., Porter, E., Robinaugh, D. J., Marks, E. H., Marques, L. M., Otto, M. W., Pollack, M. H., \& Simon, N. M. (2012). Mood regulation and quality of life in social anxiety disorder: An examination of generalized expectancies for negative mood regulation. Journal of Anxiety Disorders, 26(3), 435-441. https://doi.org/10.1016/j.janxdis.2012.01.004

Thoemmes, F. (2015). Reversing arrows in mediation models does not distinguish plausible models. Basic and Applied Social Psychology, 37(4), 226-234. https://doi.org/10.1080/01973 533.2015.1049351

UlaşanÖzgüle, E. T., \& Sümer, N. (2017). Emotional regulation and psychological adjustment in adolescence: Turkish adaptation of the emotion regulation scale. Türk Psikoloji Yazlları (turkish Psychological Articles), 20, 5-22.

Werner, K. H., Goldin, P. R., Ball, T. M., Heimberg, R. G., \& Gross, J. J. (2011). Assessing emotion regulation in social anxiety disorder: The emotion regulation interview. Journal of Psychopathology and Behavioral Assessment, 33(3), 346-354. https://doi. org/10.1007/s10862-011-9225-x

Wiedermann, W., \& Li, X. (2021). Direction dependence analysis. https://www.ddaproject.com/. Accessed 15 May 2021.

Wiedermann, W., Li, X., \& von Eye, A. (2019). Testing the causal direction of mediation effects in randomized intervention studies. Prevention Science, 20, 419-430. https://doi.org/10.1007/ s11121-018-0900-y 
Wiedermann, W., Reinke, W. M., \& Herman, K. C. (2020). Prosocial skills causally mediate the relation between effective classroom management and academic competence: An application of direction dependence analysis. Developmental Psychology, 56(9), 1723-1735. https://doi.org/10.1037/dev0001087

Wiedermann, W., \& Sebastian, J. (2019). Direction dependence analysis in the presence of confounders: Applications to linear mediation models using observational data. Multivariate Behavioral Research, 55(4), 495-515. https://doi.org/10.1080/ 00273171.2018 .1528542

Wiedermann, W., \& von Eye, A. (2015). Direction of effects in mediation analysis. Psychological Methods, 20, 221-244. https://doi. org/10.1037/met0000027

Wong, Q. J. J., Gregory, B., Gaston, J. E., Rapee, R. M., Wilson, J. K., \& Abbott, M. J. (2017). Development and validation of the core beliefs questionnaire in a sample of individuals with social anxiety disorder. Journal of Affective Disorders, 207, 121-127. https://doi. org/10.1016/j.jad.2016.09.020

Zaki, J., \& Williams, W. C. (2013). Interpersonal emotion regulation. Emotion, 13(5), 803-810. https://doi.org/10.1037/a0033839

Zigmond, A. S., \& Snaith, R. P. (1983). The hospital anxiety and depression scale. Acta Psychiatrica Scandinavica, 67(6), 361370. https://doi.org/10.1111/j.1600-0447.1983.tb09716.x

Publisher's Note Springer Nature remains neutral with regard to jurisdictional claims in published maps and institutional affiliations. 